\title{
CARACTERIZAÇÃO DAS PLANTAS MEDICINAIS E MEDICAMENTOS FITOTERÁPICOS PARA TRATAMENTO DA OSTEOPOROSE UTILIZADOS NO BRASIL
}

\author{
Marcia Alessandra Arantes Marques ${ }^{1}$ \\ Daniely Alves Lima ${ }^{2}$ \\ Carlos Eduardo Andreotti ${ }^{2}$ \\ Arquimedes Gasparotto Junior ${ }^{4}$ \\ Emerson Luiz Botelho Lourenço ${ }^{5}$
}

\begin{abstract}
MARQUES, M. A. A.; LIMA, D. A.; ANDREOTTI, C. E.; GASPAROTTO JUNIOR, A.; LOURENÇO, E. L. B. Caracterização das plantas medicinais e medicamentos fitoterápicos para tratamento da osteoporose utilizados no Brasil. Arq. Cienc. Saúde UNIPAR, Umuarama, v. 20, n. 3, p, 183-188, set./dez. 2016.
\end{abstract}

\begin{abstract}
RESUMO: A osteoporose é caracterizada por desordem do sistema esquelético caracterizada por baixa massa óssea e deteriorização da microarquitetura do tecido ósseo levando a fragilidade óssea com aumento da suscetibilidade a fraturas. Considerada a doença osteometabólica mais comum, de distribuição global, pode comprometer ambos os sexos, diferentes idades ou raças. As consequências relacionadas a essas doenças são as fraturas vertebrais verificadas em aproximadamente $20 \%$ das mulheres acima de 65 anos em pós-menopausa. Além disso, a consequência dessa fragilidade óssea pode levar a consequências graves como dor, deformidades, sequelas, restrições motoras e morte precoce, portanto, associada com alta morbi/mortalidade. Desta forma, sabendo que as medicações utilizadas para o tratamento da osteoporose não são isentas de risco (por exemplo, aumento do risco de tromboses, câncer de mama/ ovário/ endométrio, lesões musculares, entre outras), foi realizado uma revisão bibliográfica com o intuito de avaliar se existem plantas medicinais que possam ter efeitos ósseo protetores com menor número de efeitos adversos em relação aos fármacos sintéticos. Em síntese, verificou-se que os fármacos sintéticos podem acarretar prejuízos à saúde do paciente a longo prazo e que as plantas medicinais ou fitoterápicos utilizadas no Brasil apresentam estudos científicos com menores efeitos colaterais e, portanto, são uma alternativa viável para o tratamento e prevenção da osteoporose, visto que a Politica Nacional de Práticas Integrativas e Complementares na Atenção Básica sustenta esta tendência mediante subsídios como a Farmacopeia Brasileira e o Memento Fitoterápico.
\end{abstract}

PALAVRAS-CHAVE: Brasil. Fitoterapia. Medicamentos Fitoterápicos. Osteoporose.

\section{CHARACTERIZATION OF MEDICINAL PLANTS AND HERBAL MEDICINES FOR THE TREATMENT OF OSTEOPOROSIS USED IN BRAZIL}

\begin{abstract}
Osteoporosis is characterized by skeletal system disorder, consisting of low bone mass and deterioration of the microarchitecture of the bone tissue, leading to bone fragility with increased susceptibility to fractures. Considered the most common osteometabolic disease worldwide, it affects both sexes, different ages and races. The consequences of these diseases are vertebral fractures in approximately $20 \%$ of postmenopausal women over 65 years of age. In addition, the consequence of this bone fragility may lead to serious consequences, such as pain, deformities, sequelae, motor restrictions and early death, being therefore associated with high morbidity/mortality. Therefore, knowing the medication used in the treatment of osteoporosis are not free of risk (for example, increased risk of thrombosis, breast/ovarian/endometrial cancer, muscle injuries, among others), a bibliographical review was made with the intent of evaluating whether there are medicinal plants that may have protective effects on the bones with a decreased number of side effects in relation to synthetic medication. In summary, we found that synthetic drugs may cause long-term health damage in the patient, and that herbal or medicinal plants used in Brazil present scientific studies with lower side effects. They are, therefore, a viable alternative for the treatment and prevention of osteoporosis, since this trend is supported by the National Policy of Integrative and Complementary Practices in Primary Care through subsidies, such as the Brazilian Pharmacopoeia and the Phytotherapeutic Memento.
\end{abstract}

KEYWORDS: Brazil. Herbal Medicines. Osteoporosis. Phytotherapy.

\section{Introdução}

A osteoporose é definida por desordem do sistema esquelético caracterizada por baixa massa óssea e deteriorização da microarquitetura do tecido ósseo levando a fragilidade óssea com aumento da suscetibilidade à fraturas (HOLROYD; DENNISON; COOPER, 2011). Considerada a doença osteometabólica mais comum, de distribuição global, pode comprometer ambos os sexos, diferentes idades ou raças. As fraturas, consequência dessa fragilidade óssea, po- dem levar à consequências graves como dor, deformidades, sequelas, restrições motoras e morte precoce, portanto, associada com alta morbi/mortalidade. Com o aumento da expectativa de vida, a osteoporose vem aumentando sua incidência. Sabe-se que apesar das várias etiologias para a osteoporose sem dúvida a mais importante é o envelhecimento. No Brasil, em um estudo realizado com idosos, foi constatado que as lesões provocadas pela osteoporose são responsáveis pela prevalência dos óbitos nos indivíduos com idade superior a 60 anos. Os números relacionados a estas evidências mos-

DOI: https://doi.org/10.25110/arqsaude.v20i3.2016.5870

${ }^{1}$ Universidade Paranaense, Médica, aluna do programa de Mestrado Profissional em Plantas Medicinais e Fitoterápicos na Atenção Básica, 87502-210, Umuarama, PR, Brasil. doutoramarciamarques@gmail.com

${ }^{2}$ Universidade Paranaense, aluna do curso de graduação Farmácia, 87502-210, Umuarama, PR, Brasil. danielyalvesdelima@hotmail.com

${ }^{3}$ Universidade Paranaense, aluno do curso de graduação Medicina Veterinária, 87502-210, Umuarama, PR, Brasil. caduandreotti@yahoo.com.br

${ }^{4}$ Universidade Federal da Grande Dourados, Faculdade de Ciências da Saúde, Cx. Postal 533, 79804-970 Dourados, MS, Brasil. arquimedesgasparotto@ gmail.com

${ }^{5}$ Universidade Paranaense, professor do programa de Mestrado Profissional em Plantas Medicinais e Fitoterápicos na Atenção Básica, 87502-210, Umuarama, PR, Brasil. emerson@unipar.br 
tram que ocorrem aproximadamente 127 mil lesões fatais e 730 hospitalizações nos hospitais públicos. Estes dados acarretam o aumento da mortalidade, redução da qualidade vida e um aumento dos gastos para o setor da saúde (ARANTES; SILVA; LAZARETTI-CASTRO, 2010; PINHEIRO et al., 2010). De acordo com a Organização Mundial de Saúde, 1,7 milhões de fraturas de quadril ocorrem no mundo todo. Considerando as mudanças nos padrões demográficos da população os números poderão alcançar 6 milhões até 2050 (YAZDANI et al., 2011). Desde o nascimento até em torno dos 40 anos, a massa óssea é constantemente aumentada sendo dependente de fatores intrínsecos e extrínsecos ao corpo humano onde a formação óssea é maior que a reabsorção óssea. Após os 40 anos, ocorre uma perda de massa óssea de 0,5 a $1 \%$ ao ano e nas mulheres no período pós-menopausa essa perda aumenta em mais $2 \%$ ao ano. Portanto, as mulheres após os 50 anos de idade estão mais predispostas à doença e estima-se que uma em cada três mulheres, atualmente, deve sofrer algum tipo de fratura relacionada à osteoporose, dentre elas as fraturas vertebrais, colo de fêmur e as do antebraço. (RADOMINSKI et al., 2004).

O osso não é uma estrutura inerte, mas sim uma estrutura em estado de remodelação óssea permanente. Assim, o desacoplamento da remodelação óssea está relacionado à fisiopatologia da osteoporose, com aumento da reabsorção, e redução da formação óssea. A remodelação óssea é definida como um processo relacionado entre a reabsorção óssea e formação óssea (AMADEI et al., 2006; (ARANTES; SILVA; LAZARETTI-CASTRO, 2010). Os osteoblastos após a diferenciação em osteoclastos promovem a reabsorção óssea e a remodelação no mesmo local onde se encontra a ondulação óssea. Osteoblastos desempenham um papel chave na secreção do mediador para a osteoclastogênese, o ativador de receptor de fator nuclear (RANK) e ligante (RANKL), se liga a este receptor (RANK) na membrana plasmática de precursores de osteoclastos, e estimulam a diferenciação de pré - osteoclastos para osteoclastos maduros. RANKL e citocinas denominadas fatores estimuladores de colônia de macrófagos (MCSF) são desensibilizadas de formas distintas por vários fatores osteoclastogênicos, como o hormônio da paratireoide (PTH), PTH peptídeo relacionado e prolactina. Além disso, para contrabalancear a ação de RANKL, os osteoblastos sintetizam e secretam osteoprotogerina (OPG), um receptor solúvel capaz de inibir a interação RANK-RANKL e a osteoclastogênese. Na presença de osteoclastos ativados, a reabsorção óssea se inicia com a dissolução de componentes orgânicos e inorgânicos por ácido hidroclórico, catepsina $\mathrm{K}$ e proteases lisossomais de osteoclastos (WONGDEE; CHAROENPHANDHU, 2011).

Existem fatores determinantes sobre a determinação da massa óssea que se dividem em dois grupos. Aqueles considerados não modificáveis como: genética (gênero/ idade) e pico de massa óssea, e aqueles modificáveis como: estilo de vida (tabagismo/ etilismo); exercícios físicos; alterações hormonais, deficiências nutricionais (magnésio, fósforo, cálcio, vitamina $K$, vitamina $D$ ), doenças crônicas (doenças inflamatórias intestinais; doenças inflamatórias autoimunes; doenças endócrinas; doenças renais; neoplasias/ doenças ósseas) e uso de medicamentos (principalmente corticosteroides, anticonvulsivantes, quimioterápicos alguns antidepressivos e bloqueadores H2). Portanto, as alterações relacionadas à osteoporose têm caráter multifatorial e, desta forma, torna-se necessário o diagnóstico da etiologia para que se possa dar o tratamento adequado. Dentre as causas hormonais, uma das mais importantes é a menopausa, estado fisiológico de todas as mulheres, que pode iniciar-se após os 40 anos sendo a responsável pela a maior parte dos casos de osteoporose. A diminuição dos hormônios sexuais esteroidais leva um aumento da formação de osteoclastos e osteoblastos na medula óssea com aumento na produção de citocinas, incluindo IL-6 (interleucina -6), TNF (fator de necrose tumoral), IL-1(interleucina-1) e de fatores estimuladores de colônia de macrófagos (MCSF) quais mediam as respostas osteoclastogenesis e osteoblastogenesis (TREMOLLIERES; RIBOT, 2010).

Assim, o tratamento da osteoporose também é multifatorial. Mudança no estilo de vida como: alimentação equilibrada com quantidades suficientes de cálcio, vitamina d e outros minerais; assim como estímulo a atividade física e abandonar vícios (tabagismo/etilismo) irão influenciar positivamente na saúde óssea. Em pacientes selecionados opta-se por tratamento medicamentoso. Essas medicações não são isentas de risco. O uso de cálcio e vitamina D ainda é controverso. Alguns estudos sugerem que o consumo de cálcio pode levar à calcificações vasculares levando a risco cardiovascular aumentado apesar de ambos serem importantes para qualidade óssea (YANG, et al., 2016). Os bisfosfonatos, os mais utilizados, estão associados com aumento da incidência de osteonecrose de mandíbula, rabdomiólise, fraturas atípicas, entre outros. As medicações hormonais (reposição hormonal e moduladores seletivos do receptor de estrogênio) também não são isentas de risco. Doenças como neoplasia mamária, endométrio, tromboses já estão bem documentadas (DEAL; ABELSON, 2011).

Outras medicações como PTH o anticorpo monoclonal anti-RanKL (Ac anti-RANKL) são medicamentos com risco aumentado de sarcoma e celulite de membros inferiores, respectivamente e apresentam uma boa resposta, porém tem um alto custo no tratamento. Considerando que as medicações não podem ser utilizadas continuamente devido principalmente aos seus efeitos adversos, torna-se importante o conhecimento sobre a utilização de plantas medicinais para prevenir a perda óssea, na prevenção e/ou no tratamento da osteoporose, conferindo uma segurança no seu uso a longo prazo, adiando, portanto, as medicações alopáticas e consequentemente seus efeitos colaterais (DEAL; ABELSON, 2011).

Dentro dessa prática, foi pactuada na Comissão Intergestores Tripartite, aprovada pelo Conselho Nacional de Saúde no ano de 2005 e publicada por meio de Portaria GM $n^{\circ}$ 971, de 03 de maio de 2006, a Política Nacional de Práticas Complementares e Integrativas do SUS (PNPIC) que propôs a inclusão das Plantas Medicinais e dos Fitoterápicos como opções terapêuticas no Sistema Público de Saúde (MINISTÉRIO DA SAÚDE, 2012). Essa política traz dentre suas diretrizes a elaboração da Relação Nacional de Plantas Medicinais e de Fitoterápicos de interesse ao SUS (RENISUS) em 2009, que apresentou uma lista com 71 diferentes espécies vegetais de interesse ao SUS visando o acesso seguro e o uso racional de plantas medicinais e fitoterápicos pela população (MINISTÉRIO DA SAÚDE, 2014). Desta forma, é importante conceituar as diferenças entre os termos; 
Plantas Medicinais, Fitoterápicos e fitoterapia. De acordo com a definição proposta pela ANVISA, as plantas medicinais são aquelas capazes de aliviar ou curar enfermidades e têm tradição de uso como remédio em uma população ou comunidade. Para usá-las, é preciso conhecer a planta e saber onde colhê-la e como prepará-la. Quando a planta medicinal é industrializada para se obter um medicamento, tem-se como resultado o fitoterápico. Já o termo fitoterapia foi dado à terapêutica que utiliza os medicamentos cujos constituintes ativos são plantas ou derivados vegetais, e que tem a sua origem no conhecimento e no uso popular (MINISTÉRIO DA SAÚDE, 2012). Nessa lista contém as seguintes plantas abordadas abaixo: Glycine max (soja); Trifolium pratense (trevo vermelho), Curcuma longa (açafrão) e somente uma não está incluída nessa lista, sendo ela a Cimicifuga racemosa (cohosh preto).

Desta forma, o objetivo dessa revisão é mostrar que existem medicamentos fitoterápicos que podem ser utilizadas para o tratamento da osteoporose, pois já existem estudos que podem fundamentar esse uso em pacientes selecionados.

\section{Desenvolvimento}

Foi realizado um estudo exploratório, descritivo, de natureza qualitativa, apresentado sobre a forma de revisão de literatura e que teve como questão norteadora: Quais são as Plantas Medicinais e ou fitoterápicos que podem ser utilizados no Brasil para o tratamento da osteoporose?

Durante a realização desta revisão de literatura, a busca por publicações científicas foi realizada no período compreendido entre agosto e outubro de 2016 por meio de consultas realizadas por intermédio da internet e publicações científicas disponibilizadas em periódicos online, a partir do acervo da Scientific Electronic Library Online (SCIELO), Google Acadêmico e acervo pessoal dos autores. A busca por publicações ocorreu a partir dos seguintes critérios de inclusão: artigo completo publicado no período de janeiro/ 2002 à outubro/ 2016 ; disponível no idioma português e inglês;

Para localização das publicações utilizou-se os seguintes descritores cruzados: 1) Plantas medicinais, 2) fitoterápicos; 3) hormônios, 4) osteoporose; e 5) Riscos, na forma para "todos os índices" e "todas as fontes", disponível no banco de dados da SCIELO e com busca de modo integrado no Google acadêmico. A pesquisa gerou 521 artigos. A análise e discussão dos dados obtidos são explicitadas a seguir, respeitando-se a autoria das produções.

Apesar de existirem várias plantas, que na cultura popular podem ser utilizadas para a prevenção e tratamento da osteoporose, estudos sobre essas plantas ainda são escassos. Ao contrário dos fármacos sintéticos que são baseados em um único produto químico, as plantas medicinais exibem vários benefícios através da ação aditiva e/ou sinérgica dos numerosos componentes ativos atuando em múltiplos alvos associados com o processo fisiológicos. Desde a antiguidade o homem se utiliza de plantas com fins medicinais, para tratamento ou prevenção de doenças. Na década de 1990, a Organização Mundial de Saúde (OMS) divulgou que 65-80\% da população dos países em desenvolvimento dependiam das plantas medicinais como única forma de acesso aos cuidados básicos de saúde (VEIGA; PINTO; MACIEL, 2005). Apesar da evolução da medicina a partir da segunda metade do sécu- lo XX, existe dificuldade no acesso, que compreende desde $\mathrm{o}$ atendimento hospitalar à obtenção de exames e até medicamentos. Além disso, há também os efeitos colaterais dessas medicações que, em algumas vezes, é o suficiente para descontinuação do tratamento.

Apesar da grande propaganda atual para se utilizar plantas medicinais, a maioria das suas supostas propriedades farmacológicas anunciadas não possuem validade científica, por não terem sido investigadas ou por não ter suas ações comprovadas em testes científicos (VEIGA; PINTO; MACIEL, 2005). A maior parte dessas plantas é vendida em feiras ou lojas de produtos naturais sem controle de qualidade adequado e sem normas que garantem a procedência e, portanto a certificação (VEIGA; PINTO; MACIEL, 2005). Existe a cultura popular de que as plantas medicinais são atóxicas e que podem ser administradas concomitantemente com outros fármacos, o que não é verdade. Os efeitos adversos dos fitomedicamentos, possíveis adulterações e toxidez, bem como a ação sinérgica (interação com outras drogas) ocorrem comumente (VEIGA; PINTO; MACIEL, 2005). Portanto, estudos clínicos e toxicológicos dessas plantas assim como suas interações farmacológicas são necessários. Já existem plantas estudadas, validadas e aprovadas para o tratamento de síndrome climatérica, chamados fitoestrogênios, que demonstram efeito protetor aos ossos. A princípio, esse tratamento vem associado à terapia de reposição hormonal que apresenta ação importante no alívio dos sintomas do climatério com efeitos positivos na massa óssea como uma alternativa à medicação alopática. Portanto, a terapia com plantas medicinais visa minimizar os efeitos colaterais e diminuindo o custo do tratamento das fraturas e suas complicações.

\section{As plantas medicinais que podem ser utilizadas para tra- tamento da osteoporose no Brasil estão descritas abaixo:}

Glycine max (Soja). O extrato rico em isoflavonas é indicado para tratamento de sintoma do climatério. (BALBINO; DIAS, 2010). Essa planta é a mais estudada, dentro do grupo dos fitoestrogênicos, principalmente como isoflavonas como glicosídeos (genisteína e daidzeína) que são hidrolisadas no intestino formando agliconas seletivas do receptor estrogênico (RE). (JIA et al., 2012; TAYLOR, 2015, LOPEZ-GUTIERREZ et al., 2014). Na mucosa intestinal, a microflora metaboliza a daidzeína em S-quol que apresenta melhor atividade estrogênica e alta afinidade para o RE que a daidzeína levando a efeitos estrógeno-símile melhorando os sintomas do climatério (DIETZ et al., 2016). A genisteína estimula a formação óssea, inibindo a reabsorção e prevenindo a perda de massa óssea em modelos de ratas ovariectomizadas. Em mulheres na pós-menopausa, estudos demonstram que a genisteína foi efetiva na prevenção da perda de massa óssea (MORABITO et al., 2002; MARINI et al., 2007).

As isoflavonas da soja têm, portanto, efeitos benéficos na densidade mineral óssea, marcadores de tunover ósseo e na resistência óssea na mulher pós-menopausa sendo que a dieta contendo $22 \%$ de proteínas de soja pode ser tão efetiva quando a administração de estrogênio diário na supressão da perda óssea induzida por ovarectomia (JIA et al., 2012). Um estudo mostrou que as isoflavonas da soja promovem a proliferação e diferenciação dos osteoblastos, diminuindo acentuadamente os níveis RANKL, aumentando os níveis de 
OPG em células osteoblásticas resultando em ativação da via $\beta$-catenina/ Wnt (YU et al., 2015). A associação das isoflavonas de soja com $\beta$-caroteno também mostrou benefício para a formação óssea com manutenção de um equilíbrio positivo na remodelação óssea, por indução da diferenciação de osteoblastos (NISHIDE et al., 2015). Segundo DIETZ et al (2016) evidências sugerem que os efeitos da isoflavona na saúde óssea pode variar em função da idade e de acordo com REINWALD; WEAVER (2006) sendo mais efetiva em mulheres jovens antes que ocorra a perda dos receptores estrogênicos observada na menopausa. Outros estudos mostraram que a proteína de soja ou isoflavonas de soja enriquecida não são formas efetivas na prevenção da perda de massa óssea ou fraturas nas mulheres na Peri e na pós menopausa (ALEKEL et al., 2010). Segundo a ANVISA (2016), esse fitoterápico é contraindicado para menores de12 anos e/ou gestantes; em pacientes com histórico de hipersensibilidade e alergia a qualquer um dos componentes do fitoterápico e orientam que pode provocar distúrbios gastrointestinais leves como constipação, flatulência e náusea. GRANDI (2014) descreve que a Glycine max pode aumentar a formação de cálculos renais por oxalato de cálcio (por reduzir a absorção de íons de ferro, cálcio e zinco). No mercado brasileiro pode ser encontrada com o nome comercial Piascledine ${ }^{\circledR}$, uma associação com Persea gratissima (ANVISA, 2016), e Isoflavine $\AA$, entre outras .

Trifolium pratense (Trevo vermelho). Essa planta contém isoflavonas estrogênicas, assim como a soja, sendo as principais a biochanina proestrogênica e formononetina (BOOTH et al., 2006). Os precursores metoxi são desmetilados pelo isozimas do citocromo P450 no intestino e no fígado em genisteína e daidzeína seletivas para o RE (DIETZ et al., 2016) que são efetivas na perda óssea induzida por ovarectomia, provavelmente pela redução no tunover ósseo da reabsorção óssea (CIRCOSTA et al., 2006; BECK; ROHR; JUNGBAUER, 2005). Além disso, estudos recentes demonstram que o seu extrato enriquecido aumentou os níveis de fosfatase alcalina óssea e de estradiol de forma significativa em ratas ovariectomizadas assim como aumento significativo da densidade mineral óssea. (KANG et al., 2015). Devido aos seus efeitos estrogênicos, seu uso deve ser contraindicado em gestantes e crianças. No mercado brasileiro pode ser encontrada com o nome comercial Climadil ${ }^{\circledR}$ com indicação em bula para o alívio dos sintomas da menopausa ocorrendo uma melhora da incidência e severidade das ondas de calor, melhorando o bem estar e após sua utilização por um ano foi observado a diminuição do grau de perda óssea em mulheres pré e peri-menopausadas.

Cimicifuga racemosa (cohosh preto). É utilizada na medicina tradicional, para sintomas do climatério além de dismenorréia e dores do parto (JIA et al., 2012). Seu efeito não é atribuído aos efeitos estrogênicos e sim, ao mecanismo de ação que envolve a modulação do sistema serotoninérgico similarmente aos antidepressivos e, portanto, não altera o padrão hormonal associado à menopausa (JIA et al., 2012; WUTTKE, et al., 2014). Os componentes ativos não são conhecidos, embora N-metil serotonina tem sido identificada com potencial agonista do receptor serotoninérgico (DIETZ et al., 2016) No osso atua aumentando significativamente a densidade mineral óssea trabecular na metáfise da tíbia e melhora a taxa de diferenciação de OPG para RANK L em osteoblastos humanos normais (WUTTKE; SEIDLOVA-WUTTKE; GORKOW, 2003). QIU et al (2007) demonstrou que um glicosídeo triperteno isolado bloqueou potencialmente a osteoclatogenesis, in vitro, induzida por ambos RANKL ou TNF- $\alpha$. É uma medicação contraindicada para pacientes com história de tumor estrogênio dependente ou câncer endometrial, na gravidez e amamentação e deve ser usado com cautela por pessoas alérgicas ao ácido acetilsalicílico e a outros salicilatos (CASTRO et al., 2006). Além disso, há um relato de caso de hepatite fulminante com o seu uso (LYNCH; FOLKERS; HUTSON, 2006) indicando que um acompanhamento laboratorial hepático deve ser realizado quando essa medicação é administrada. No mercado brasileiro pode ser encontrada com o nome comercial Aplause $\mathbb{R}$ com indicação em bula para Alívio dos sintomas do climatério, como rubor, ondas de calor, suor excessivo, palpitações e alterações depressivas de humor.

Curcuma longa (Açafrão). Utilizado, na cultura popular, como condimento alimentar e para o tratamento de sintomas de flatulência, icterícia, alterações menstruais, hematúria, hemorragia, cólica e anit-inflamatório (LABBAN, 2014). Contém bioativo curcuminóides fenólicos, avaliados quanto aos efeitos ósseo-protetor num modelo animal (ratas ovariectomizadas) mostrou que o mesmo não evitou a perda óssea, no entanto, quando enriquecido preservou o número e conectividade das trabéculas sugerindo que a cúrcuma pode ter efeitos ósseos protetores dependendo da concentração do extrato (WRIGHT et al., 2010). Outro estudo mostrou que a curcumina pode evitar uma maior deterioração da estrutura óssea e produzir mudanças benéficas na remodelação óssea em ratos transgênicos APP / PS1 que são suscetíveis à osteoporose (YANG et al., 2011). Estudos recentes, indicam conclusivamente que Calebin A, derivado da cúrcuma longa, diminui a osteoclastogênese induzida por RANKL e pelas células tumorais através da supressão do NF-kB via (TYAGI et al., 2016). Essa medicação deve ser evitada em pacientes com sensibilidade ou alergia a curcumina ou qualquer componente da Curcuma longa L. (GRANDI, 2014) e em pacientes que estejam fazendo uso de medicações que alterem suas características de coagulação como: antiagregantes plaquetários, anticoagulantes, heparina de baixo peso molecular e agentes trombolíticos; pacientes com riscos de obstrução de vias biliares ou que tenham cálculos biliares; pacientes com úlceras estomacais e hiperacidez do estômago; crianças; gestantes e lactantes (GRANDI, 2014). No mercado brasileiro pode ser encontrada com o nome comercial Motore $\AA$, fitoterápico com indicação em bula, para o tratamento de osteoartrite e artrite reumatóide com ação anti-inflamatória e antioxidante.

\section{Conclusão}

Apesar da imensa biodiversidade brasileira e do conhecimento popular sobre as plantas medicinais, representada pelos raizeiros, ainda existe pouca informação científica sobre outras plantas que poderiam ser utilizadas para prevenção e tratamento da osteoporose. Os estudos já realizados, nas plantas citadas nesse artigo, mostraram ser promissoras no tratamento da osteoporose. Deve-se, portanto, incentivar o uso de tratamento natural/fitoterápico levando em consideração uma série de fatores importantes para sua prescrição 
como: a idade do paciente, gravidade da doença, tempo de tratamento; tolerabilidade das medicações alopáticas, interações e contraindicações medicamentosas (inclusive com hormônios sexuais sintéticos). Assim, é importante que as políticas públicas existentes sejam fortalecidas no âmbito da criação de estudos clínicos multicêntricos que orientem um protocolo apropriado às individualidades de cada paciente, a partir da medicina tradicional com ênfase nas plantas medicinais de interesse do SUS.

\section{Referências}

ALEKEL, D. L. et al. The soy isoflavones for reducing bone loss (SIRBL) study: a 3-y randomized controlled trial in postmenopausal women. Am J Clin Nutr. v. 91, n. 1, p. $218-230,2010$

AMADEI, S. U. et al. A influência da deficiência estrogênica no processo de remodelação e reparação óssea. J Bras Patol Med Lab. v. 42, p. 5 - 12, 2006.

ANVISA. Memento Fitoterápico: Farmacopéia Brasileira. 1 ed. Agência Nacional de Vigilância Sanitária: Brasília, 2016.

APLAUSE: Cimicifuga racemosa. Regina Helena Vieira de Souza Marques. São Paulo: Marjan Indústria e Comércio Ltda, 2013. Bula de remédio.

ARANTES, H. P., SILVA, A. G., LAZARETTICASTRO, M. Bisphosphonates in the treatment of metabolic bone diseases. Arq Bras Endocrinol Metabol. v. 54, p. 206-212, 2010.

BALBINO, E. E. ; DIAS, M. F. Farmacovigilância: um passo em direção ao uso racional de plantas medicinais e fitoterápicos. Revista Brasileira de Farmacognosia, 2010.

BECK, V.; ROHR, U.; JUNGBAUER, A. Phytoestrogens derived from red clover: an alternative to estrogen replacement therapy? J Steroid Biochem Mol Biol. p. 499518,2005

BOOTH, N. L. et al. The chemical and biologic profile of a red clover (Trifolium pratense L.) phase II clinical extract. J Altern Complement Med. v. 12, n. 2, p. 133-9, 2006.

BRASIL. Ministério da Saúde Plantas de Interesse ao SUS. Portal da saúde [online]. 2014. Disponível em: $<$ http:// portalsaude.saude.gov.br/index.php/o-ministerio/principal/ leia-mais-o-ministerio/466-sctie-raiz/daf-raiz/ceaf-sctie/ fitoterapicos-cgafb/12-fitoterapicos/15824-consultapublica-n-28-2014. Acesso em: 08.11. 2016.

BRASIL. Ministerio da saude. Anvisa. Disponível em: < http://www.anvisa.gov.br/medicamentos/fitoterapicos/ poster_fitoterapicos.pdf. Acesso em: 12 nov. 2016

BRASIL. Ministério da Saúde. Práticas integrativas e complementares: plantas medicinais e fitoterapia na Atenção Básica/Ministério da Saúde. Secretaria de Atenção à Saúde.
Departamento de Atenção Básica. - Brasília: Ministério da Saúde, 156 p. n. 31. 2012.

CASTRO, T. A; CAMARGO, A. L; HEINECK, I.; FISCHER, M. I. Cimicifuga. Boletim Informativo do CIM-RS, 2006. Disponível em: < http:// www.ufrgs.br / boletimcimrs / cimicifuga.pdf. Acesso em: 14 out. 2016

CIRCOSTA, C. et al. Effects of isoflavones from red clover (Trifolium pratense) on skin changes induced by ovariectomiy in rats. Phytotherapy Research. v. 20, n. 12, p. 1096-1099, 2006.

CLIMADIL: Trifolium pratense. Regina Helena Vieira de Souza Marques. São Paulo: Marjan Indústria e Comércio Ltda, 2013. Bula de remédio.

DEAL, C. L.; ABELSON, A. G. Management of osteoporosis. In: Hochberg, M. C. et al. Rheumatology. Philadelphia: Elsevier, p. 1975-1988, 2011.

DIETZ, B. M. et al. Botanicals and Their Bioactive Phytochemicals for Women's Health. Pharmacol Rev. v. 68, n. 4, p.1026-1073, 2016.

GRANDI, T. S. M. Tratado das plantas medicinais: Mineiras, Nativas e Cultivadas. Adaequatio Estúdio. p. $1076-1077,2014$.

HOLROYD, C.; DENNISON, E.; COOPER, C. Epidemiology and Classification of metabolic bone disease. In: Hochberg, M.C. et al. Rheumatology. Philadelphia: Elsevier, p. 1937-1944, 2011.

JIA, M. et al. Potential antiosteoporotic agents from plants: a comprehensive review. Evid Based Complement Alternat Med, 2012.

KANG, S. J. et al. Dried pomegranate potentiates antiosteoporotic and anti-obesity activities of red clover dry extracts in ovariectomized rats. Nutrient. p. 2622-2647, 2015.

LABBAN, L. Medicinal and pharmacological properties of Turmeric (Curcuma longa): A review. Int J Pharm Biomed Sci. v. 5, n. 1, p. 17-23, 2014.

LOPEZ-GUTIERREZ, N. et al. Identification and quantification of the main isoflavones and other phytochemicals in soy based nutraceutical products by liquid chromatography-orbitrap high resolution mass spectrometry. J Chromatogr A. p.125-136, 2014.

LYNCH, C.R.; FOLKERS, M. E.; HUTSON, W. R. Fulminant Hepatic Failure Associated With the Use of Black Cohosh: A Case Report. Liver Transplatation. v. 12, p. 989-992, 2006.

MARINI, H. et al. Effects of the phytoestrogen genistein on bone metabolism in osteopenic postmenopausal women: a randomized trial. Ann Intern Med. v.146, n. 12, p. 839- 
$847,2007$.

MORABITO, N. et al. Effects of genistein and hormonereplacement therapy on bone loss in early postmenopausal women: a randomized double-blind placebo-controlled study. J Bone Miner Res. v.17, n. 10, p. 1904-1912, 2002.

MOTORE: Curcuma longa. Gabriela Mallmann. Guarulhos: Aché Laboratórios Farmacêuticos S.A., 2012. Bula de remédio.

NISHIDE, Y. et al. Combined Effects of Soy Isoflavones and $\beta$-Carotene on Osteoblast Differentiation. Int $\mathbf{J}$ Environ Res Public Health. v. 12, n. 11, p. 13750-61, 2015.

PIASCLEDINE: Persea Americana/ Glycine max. Ana Paula Antunes Azevedo. França: Laboratoires Expanscience, 2016. Bula de remédio.

PINHEIRO, M. M. et al. Risk factors for recurrent falls among Brazilian women and men: the Brazilian Osteoporosis Study (BRAZOS). Cad Saude Publica. n. 26 p. 89-96. 2010.

QIU, S. X. et al. A triterpene glycoside from black cohosh that inhibits osteoclastogenesis by modulating RANKL and TNFalpha signaling pathways. Chem Biol. v.14, n. 7, p. 860-869, 2007.

RADOMINSKI, S. C. et al. Osteoporose em mulheres na pós-menopausa. Rev. Bras. Reumatol. v. 44, n. 6, 2004.

REINWALD, S.; WEAVER, C. M. Soy isoflavones and bone health: a double-edged sword? J Nat Prod. v.69, n. 3, p.450-459, 2006.

TAYLOR, M. Complementary and Alternative Approaches to Menopause. Endocrinol Metab Clin North Am. v. 44, n. 3, p. 619-648, 2015.

TREMOLLIERES, F.; RIBOT, C. Bone mineral density and prediction of non-osteoporotic disease. Maturitas. $\mathrm{v}$. 65, n. 4, p. 348-51, 2010.

TYAGI A. K. et al. Calebin A regula negativamente osteoclastogênese através da supressão da sinalização RANKL. Arch Biochem Biophy. p. 80-89, 2016.

VEIGA V.F.; PINTO, A. C.; MACIEL M. A. Plantas Medicinais: Cura segura? Quim. Nova, v. 28, n. 3, p 519528. 2005.

WONGDEE, K.; CHAROENPHANDH, N. Osteoporosis in diabetes mellitus: Possible cellular and molecular mechanisms. World J Diabetes. v. 2, p. 41-48, 2011.

WRIGHT, L. E. et al. Protection of Trabecular Bone in Ovariectomized Rats by Turmeric (Curcuma longa L.) is Dependent on Extract Composition. J Agric Food Chem, v. 58, n. 17 , p. 9498-9504, 2010
WUTTKE, W. et al. The non-estrogenic alternative for the treatment of climacteric complaints: Black cohosh (Cimicifuga or Actaea racemosa). J Steroid Biochem Mol Biol. v. 139, p. 302-310, 2014.

WUTTKE, W.; SEIDLOVÁ-WUTTKE, D.; GORKOW, C. The Cimicifuga preparation BNO 1055 vs. conjugated estrogens in a double-blind placebo-controlled study: effects on menopause symptoms and bone markers. Maturitas. v. 14, n. 1, p. 67-77, 2003.

YANG, B. et al. Calcium intake and mortality from all causes, cancer, and cardiovascular disease: the Cancer Prevention Study II Nutrition Cohort. Am J Clin Nutr. v. 103, n. 3, p. 886-94, 2016.

YANG, M. W. et al. Curcumin improves bone microarchitecture and enhances mineral density in APP/PS1 transgenic mice. Phytomedicine. p. 205-13, 2011.

YAZDANI, S., et al. Determination of clinical decision rule for estimation of bone mineral density in women. Med Princ Pract. n. 20, p. 416-421, 2011.

YU, F. et al. Soybean isoflavone treatment induces osteoblast differentiation and proliferation by regulating analysis of Wnt/ $\beta$-catenin pathway. Gene. v. 573, n. 2, p. 273-7, 2015. 